\title{
Réseaux mobiles et management : le jeu Simobiz
}

\author{
Michel Berne \\ GET-INT, 9 Rue Charles Fourier 91011 EVRY cedex, michel.berne@int-evry.fr
}

\begin{abstract}
RESUME Le jeu d'entreprise Simobiz a été développé par l'INT pour intégrer les différentes facettes de la gestion des réseaux mobiles, de l'ingéniérie technique jusqu'au management opérationnel et à la stratégie. Il permet de simuler le fonctionnement d'un marché où plusieurs opérateurs mobiles GSM et UMTS sont en concurrence. A partir d'un scénario national défini en fonction des conditions locales et des objectifs pédagogiques visés, les participants, chargés de la gestion de plusieurs opérateurs mobiles en concurrence, déploient les réseaux nécessaires à la mise en place d'offres commerciales. L'article décrit l'architecture de Simobiz, comment il est utilisé dans des dispositifs pédagogiques existants et fait le bilan de la vingtaine de sessions de formation conduites de 2002 à 2005 dans plusieurs pays avec des participants divers.
\end{abstract}

Mots clés : réseaux mobiles, stratégie, management, jeu d'entreprise, simulation.

\section{INTRODUCTION}

Le développement phénoménal des réseaux mobiles dans le monde a généré une demande de formation spécifique considérable. D'excellents outils (livres, voire logiciels) existent en matière de formation technique. Mais les défis posés par les réseaux mobiles sont loin de se limiter au problèmes de problèmes de déploiement et d'exploitation de générations successives d'équipements et de logiciels. En un mot, il faut définir des stratégies profitables dans le long terme, intégrant les aspects techniques, marketing et managériaux. Un outil de formation bien adapté dans ce genre de cas est le jeu d'entreprise, mais si de tels dispositifs existent depuis longtemps, ils sont difficiles à concevoir dans le cas des télécommunications mobiles et l'offre est limitée [1]. L'INT, institution d'enseignement supérieur comportant une école d'ingénieurs et une école de commerce, très impliquée dans le secteur des TIC, a été confrontée à ce problème rapidement. A partir de 2000, une série de projets de recherche et de développement a permis de mettre au point un jeu spécifique, Simobiz. Nous faisons dans cet article le point successivement sur l'histoire de l'outil Simobiz, sur son architecture et ses fonctionnalités, puis concluons par un bilan de ses utilisations et des enseignements qu'on peut en tirer.

\section{LE PROJET SIMOBIZ}

Le projet a été lancé au Département Sciences de gestion de l'INT en 2000 à l'initative de Jean-Michel Sahut, enseignant-chercheur auquel se sont joints un enseignant-chercheur modélisateur (Michel Berne) et un doctorant (Rastislav Kulich).

\subsection{De l'idée à la maquette}

L'équipe d'origine a obtenu deux contrats de développement en 2000-2001: un contrat du GET dit « incitatif » regroupant l'INT, l'ENSTBr et l'Université de Zilina (Slovaquie) a permis de fixer les grandes lignes et d'asseoir scientifiquement la modélisation. Un deuxième contrat signé par l'INT avec France Télécom R\&D a débouché sur la création d'une maquette entièrement fonctionnelle au bénéfice de cette dernière entité.

\subsection{De la maquette à la commercialisation}

Sur la base des résultats obtenus, un nouveau logiciel a été développé par l'équipe de départ et validé par son utilisation par des étudiants de l'INT au printemps 2002. Depuis, des améliorations constantes ont été apportées, se traduisant en gros par une refonte annuelle partielle du logiciel. En 2003, la signature d'un contrat avec l'Union Internationale des Télécommunications a nécessité de nouveaux développements pour permettre un déploiement dans plusieurs régions du monde (Afrique, Moyen-Orient à ce jour; Amérique Latine très bientôt)

\subsection{Leçons tirées}

La première des leçons est que la construction d'un jeu d'entreprise est une opération longue et difficile : pour un modélisateur expérimenté, il est relativement rapide et aisé de concevoir le modèle de base (encore qu'il faille, dans ce cas, intégrer les relations complexes entre mobiles et réseaux sur les plans techniques et commerciaux !). Passer du stade de maquette à celui d'un produit ergonomique et stabilisé est un vrai défi, qui a pris plus d'un an à l'équipe de développement, et les efforts à déployer ne peuvent se justifier que si le produit a une durée de vie suffisante.

Malheureusement, l'évolution extrêmement rapide du secteur des mobiles oblige à une mise à jour perpétuelle. Ainsi, Simobiz intégrait l'UMTS alors même qu'aucun réseau $3 \mathrm{G}$ n'existait vraiment. La modélisation de la $3 \mathrm{G}$ s'est affinée progressivement, au fur et à mesure que les réseaux ont été déployés et les offres commercialisées.

Donc la capacité à maintenir le logiciel est essentielle, pour que les animateurs du jeu puissent l'adapter aux évolutions techniques et commerciales, comme à la diversité des situations locales. Le choix d'une solution logicielle «low-tech» a permis à chaque animateur de 
Simobiz de créer ses propres versions sans lourdeur inutile.

\section{L'ARCHITECTURE DE SIMOBIZ}

Simobiz est un logiciel modulaire qui permet de simuler le développement des réseaux et des marchés nationaux de télécommunications mobiles par périodes d'un an. Le cas typique comporte trois entreprises de télécommunications mobiles (qui possèdent chacun au moins un réseau). Les participants à la formation sont affectés à une équipe (une par opérateur) et gèrent leur réseau et leur «entreprise » en concurrence avec les deux autres équipes. Cela signifie qu'ils prennent toutes les décisions techniques et managériales d'un opérateur : prix, investissements, embauches, endettement, etc.

\subsection{Les différents blocs}

Simobiz est composé de 4 blocs principaux repris dans le schéma ci-dessous. Les opérateurs prennent des décisions de marketing (ce qu'ils veulent vendre et à quel prix). Ces décisions doivent être cohérentes avec le développement du réseau (qui constitue un autre bloc, où les opérateurs décident principalement de leurs investissements) et avec leurs ressources humaines (embauches, salaires). Les conséquences de ces décisions apparaissent dans le bloc finance.

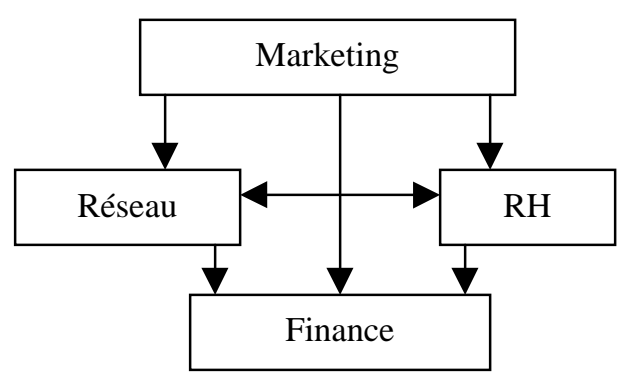

\section{fig 1: Architecture de Simobiz}

\subsection{Le scénario}

Simobiz intégre des informations de trois provenances :

- un «scénario » de jeu qui définit complètement et dès le départ l'environnement sur le plan population, topographie, règles d'ingéniérie, réglementation, structure fiscale, etc ;

- les décisions prises par les participants (opérateurs) chaque période ;

- l'historique des décisions et résultats obtenus dans les phases antérieures du jeu. Les décisions des participants sont naturellement contraintes par leurs actions antérieures (endettement, taille du réseau, etc.).

Il faut donc créer autant de scénarios que de cas nationaux. Les valeurs des paramètres du scénario sont, soit figées au départ (comme la superficie du pays), soit évolutives et modifiables si besoin, comme la popula- tion ou les prix unitaires des équipements de réseau ou encore les prix d'interconnexion. Le scénario permet de décrire des situations et des comportements très variés grâce à des choix étendus d'options.

De 2001 à 2005 plusieurs scénarios ont été développés :

«Euroland» pour les pays d'Europe occidentale;

«Visegrad » pour les pays d'Europe centrale ;

« Sahel» pour les pays du Sahel ;

«Maghreb-Machrek » pour les pays arabes.

Chaque scénario permet de décrire :

- 2 zones géographiques distinctes (démographie, économie, topographie) ;

- 4 segments de clientèle dans chaque zone ;

- 2 technologies de réseau mobile (dont le GSM).

Il est possible (comme dans le scénario consacré aux pays arabes) de démarrer avec un seul opérateur et un seul réseau et d'introduire progressivement la concurrence ; il est aussi possible de simuler des situations où on démarre avec plusieurs opérateurs, comme en Europe, avec des couvertures du territoire éventuellement partielles.

\subsection{Le bloc Marketing}

Le bloc calcule les parts de marché et le nombre de clients des opérateurs à partir des trois ensembles de données présentées ci-dessus :

- les caractéristiques de la population (démographie, revenu, répartition géographique, etc.), provenant du scénario ;

- les offres des opérateurs (mobiles, prix, publicité, etc.) ;

- l'historique des résultats de l'entreprise.

Les offres sont bâties à partir de catalogues annuels de mobiles et services réels. Par exemple, le catalogue annuel des mobiles de 2005 utilisé dans le jeu comporte 10 mobiles réels avec leurs caractéristiques principales. Dans le scénario «Euroland», les catalogues permettent le passage dans le temps du GSM au GPRS, puis à EDGE et à l'UMTS. Le bloc intègre les phénomènes de diffusion des technologies dans le temps (courbe logistique de diffusion, effet-club). Le partage du marché se fait grâce à une fonction de satisfaction des consommateurs, intégrant prix, qualité et image de marque, utilisée dans un mécanisme inspiré du modèle du logit multinomial. Cette façon de procéder a l'avantage d'être réaliste et absolument stable, deux conditions essentielles dans un jeu d'entreprise [2].

\subsection{Le bloc Réseau}

Le bloc réseau simule le fonctionnement de réseaux GSM/GPRS/EDGE d'une part et UMTS d'autre part sur la base du scénario national prédéfini et de règles d'ingéniérie simples :

- dimensionnement des réseaux à partir des prévisions de trafic des opérateurs : calcul du trafic de pointe grâce à un modèle simplifié de trafic, détermination des éléments de réseau nécessaires 
pour transporter le trafic (stations de base, MSC, etc.) ; détermination du réseau après investissement et sous contrainte de disponibilité des fréquences radio nécessaires ;

- qualité de service (couverture, congestion des réseau, maintenance, etc.) ;

- aspects réglementaires : licences, fréquences, interconnexion (in-net et off-net) ;

- MVNO (opérateurs mobiles virtuels utilisant de réseau d'un opérateur hôte).

A l'intérieur de chaque zone géographique, 3 environnements différents peuvent être définis et simulés (par exemple, en Europe : les centres des villes, les banlieues et les zones rurales).

\subsection{Le bloc Ressources humaines}

Le bloc simule la gestion du personnel, qui est réparti en six catégories ( 3 niveaux hiérarchiques pour 2 types de compétences : technique, commercial). Les opérateurs gèrent principalement les flux de personnel (embauche, licenciements, promotions, mutations d'une division à l'autre) et les salaires. Le modèle calcule un « indice de qualité du travail » et un «indice de satisfaction du personnel ». Une mauvaise gestion des ressources humaines a des répercussions négatives sur le management des réseaux et la qualité de service.

\subsection{Le bloc Finance}

Le bloc génère les comptes issus des blocs précédents : ventes, personnel, interconnexion, etc. et permet une gestion financière de base (trésorerie, dettes, dividendes). En sortie on obtient les documents classiques de gestion (bilan, compte de résultat) ainsi qu'une comptabilité analytique détaillée (coûts de réseau, coûts commerciaux).

\section{PRATIQUES PEDAGOGIQUES}

\subsection{Buts et moyens}

Les clés du succès d'un jeu d'entreprise peuvent être résumées ainsi :

- des buts pédagogiques clairs ;

- un jeu suffisamment réaliste et dynamique pour que les participants «se prennent au jeu » et en même temps « jouable », c'est à dire pas trop complexe ;

- Des allers-retours constants entre le déroulement du jeu et la réalité technique, industrielle et économique du secteur simulé ;

- Un dispositif pédagogique qui oblige les participants à prendre du recul, à synthétiser les acquis.

\subsection{Session type}

Simobiz est un jeu qui se joue par périodes réprésentant une année. Cela demande une demi-journée de travail aux participants pour prendre les décisions correspondant à une année et dépouiller les résultats. Donc en 3 jours de jeu, on peut raisonnablement simuler 5 à 6 ans de «vrai » marché. Le scénario utilisé en Europe (dit «Euroland ») a été calibré pour accélérer le temps : en 6 ans, on peut décrire toute l'évolution d'un réseau et d'un marché depuis sa mise en exploitation jusqu'à son apogée.

Dans notre pratique, une session-type prend de 3 à 5 jours. Elle peut être composée de séances bloquées ensemble ou réparties dans le temps, mais dans ce dernier cas il ne faut pas qu'elles soient trop éloignées les unes des autres. On peut aussi intégrer Simobiz à des modules de cours et alterner séances de jeu d'entreprise et séances de cours théoriques. Pour une population composée d'étudiants avancés (au niveau M1 ou M2), un schéma type peut-être le suivant :

\begin{tabular}{|c|c|}
\hline Demi-journée & Contenu \\
\hline 1 & $\begin{array}{r}\text { Découverte de Simobiz } ; \\
\text { jeu d'une période « à blanc » } \\
\text { Période } 1: \text { construction des offres } \\
\text { (marketing) }\end{array}$ \\
\hline 2 & Période $2:$ problèmes de réseau \\
\hline 3 & Période $3:$ problèmes financiers \\
\hline 4 & Période $4:$ problèmes de management \\
\hline 5 & Période $5:$ MVNO \\
\hline 6 & Conclusions \\
\hline 7 &
\end{tabular}

\section{Tableau 1 : Déroulement d'une formation}

La première séance permet la prise de contact et la constitution des équipes-entreprises. Il faut de 2 à 6 participants par équipe. Avec 3 opérateurs (qui est le nombre optimal), cela veut dire qu'un « univers » de jeu Simobiz peut accueillir de 6 à 18 participants. Au delà de 18 participants, il faut créer un « univers parallèle ». Il faut compter un animateur par univers. L'existence de jeux parallèles permet une plus grande diversité d'expériences pour les participants, qui peuvent comparer leur propre univers à d'autres.

Le passage d'une technologie à l'autre (GSM vers GPRS, EDGE, UMTS) peut-être effectué au moment que l'on désire. On peut couper le déroulement cidessus en programmant une séance pour établir un business plan consacré au déploiement de l'UMTS ; une addition intéressante est de prévoir aussi dans ce cas une séance consacrée à une enchère de licences UMTS (il suffit pour cela d'attribuer moins de licences UMTS qu'il n'y a d'opérateurs GSM).

Une caractéristique très pratique de Simobiz porte sur la possibilité de faire travailler les participants sur un bloc du modèle seulement, les décisions correspondant aux autres blocs étant prises de manière optimale par le modèle, sauf pour le bloc marketing.

Cette manière de procéder facilite l'apprentissage du système par les participants. Dans le schéma donné cidessus, on peut donc commencer par le bloc marketing (offre) et rajouter un bloc supplémentaire par séance : 
au bout de 4 séances, les participants sont entièrement responsables de toutes leurs décisions.

\subsection{Dispositif logistique}

Simobiz est un jeu peu exigeant sur le plan logistique. Le logiciel est composé de plusieurs dossiers liés Microsoft Excel, ce qui assure une très bonne portabilité. Le logiciel a été rédigé en anglais, mais tous les manuels et les sorties sont disponibles en français et en anglais en standard. Une version allégée est aussi en cours de réalisation en espagnol.

L'animateur doit disposer aussi d'une imprimante pour les sorties papier bien qu'on puisse envoyer aux équipes de participants leurs résultats sous forme de fichiers Excel. Un accès internet est également utile pour aller chercher des informations complémentaires sur le web. Les participants reçoivent un Manuel du participant (une trentaine de pages) qui inclut aussi le scénario du jeu (dit brief) et, après avoir joué chaque période, une quinzaine de pages contenant les résultats détaillés de la période.

Les animateurs reçoivent un Manuel du jeu (en 3 tomes, [3]), qui contient :

- une description du modèle avec les principales équations structurelles et comptables;

- une liste complète des paramètres du modèle ;

- un manuel pratique montrant comment conduire une session complète et en particulier utiliser le logiciel : création d'un jeu, entrée des décisions des participants, sortie des résultats en fin de période.

Les animateurs disposent aussi de plusieurs modules supplémentaires :

- d'aide à la conception des scénarios ;

- d'aide à la construction de business plans UMTS ;

- donnant les historiques de résultats du jeu ;

- et l'évaluation des performances des participants.

Sur ce dernier point, notre pratique de la notation des participants a été la suivante :

- $40 \%$ pour une évaluation multi-critère des résultats opérationnels obtenus pendant la session (résultats techniques, commerciaux, RH et financiers) ;

- $\quad 40 \%$ pour le rendu de documents produits par les participants (bilans d'étape, business plan, etc.) ;

- $20 \%$ pour la participation: un jeu ne peut pas fonctionner sans une participation active de tous !

\section{BILAN}

Le bilan d'un outil de formation doit être apprécié sur le plan quantitatif et qualitatif.

\subsection{Bilan quantitatif}

Du printemps 2002 à juillet 2005, 26 sessions de formation Simobiz ont été organisées, rassemblant plus de 450 participants originaires de 40 pays. La majorité des participants étaient des étudiants avancés (Master of Science, mastères, options de dernière année d'école d'ingénieurs et de commerce, DESS). La durée moyenne d'une session était de 3,5 jours. Les principales institutions qui en ont bénéficié sont trois des écoles du GET (INT en majorité, mais aussi ENSTBr et ENST) et les partenaires de l'INT (ITAM au Mexique, Université d'Evry Val d'Essonne, IHEC Tunis, Michigan State University aux USA).

Par ailleurs, plusieurs sessions ont été organisées pour transférer Simobiz aux Centres d'excellence de l'Union internationale des Télécommunications avec le soutien des pouvoirs publics français [4]. Sur la base de scénarios régionaux différenciés, elles ont déjà eu lieu en 2004-2005 à Dakar (ESMT), Bahrein (Batelco) et Amman (Fastlink) [5]. D'autres sessions sont prévues en Afrique francophone et anglophone, au Maroc et au Costa Rica.

Enfin, Simobiz étant extrêmement flexible, il est possible de faire fonctionner un bloc de manière indépendante. Cela a été utilisé, par exemple, pour la formation à l'INT des cadres de l'opérateur Vietnam Telecom.

\subsection{Bilan qualitatif}

Comme toute autre activité pédagogique, le déroulement et le bilan d'un jeu d'entreprise dépendent de l'interaction entre les participants, les animateurs et le dispositif de formation. Dans le cas d'un jeu, il existe par construction une grande variété de trajectoires et de pratiques qu'il faut pouvoir gérer. Les résultats de la vingtaine de jeux joués de 2002 à mi-2005 ont tous été différents, mais l'existence de scénarios structurants a permis de vérifier que, de manière diverse, les objectifs pédagogiques avaient été atteints. Autrement dit, il n'est pas important de se trouver dans une situation ou une autre - par exemple : de la congestion dans les réseaux, ou pas -, mais il est très important que tout le monde ait bien compris les implications techniques et économiques de la congestion des réseaux. Dans cette optique, il est capital de conduire des bilans intermédiaires et des séances d'analyse des résultats obtenus.

Un jeu comme Simobiz permet en outre de mener de manière aisée des simulations annexes (du type : que se serait-il passé si tel opérateur avait baissé ses prix ? ou s'il avait mal prévu le trafic dans son réseau ?). Ces simulations permettent de mettre en évidence des phénomènes complexes, comme des boucles de rétroaction entre blocs, des effets-retard, des effets de cliquet, etc. Il est ainsi possible de répondre de manière complète, chiffrée et à long terme à des questions en général presque impossibles à traiter autrement sans d'énormes simplifications comme :

- quel est le coût marginal de la minute dans le réseau?

- que se passe-t-il quand le nombre d'opérateurs varie dans le temps?

- quels sont les effets d'un manque de personnel technique?

Naturellement, la qualité des résultats fournis par Simobiz dépend de la pertinence des données d'entrée et de la cohérence du scénario. Après calibrage, un scéna- 
rio permet d'obtenir des résultats très vraisemblables d'après notre expérience.

Un processus de suivi des acquis pédagogiques a été mis en place et montre une très grande satisfaction des participants. Les principaux enseignements qu'on peut tirer des déclarations des participants en fin de formation portent sur la compréhension des points suivants :

- l'intégration des différentes fonctions dans la gestion des opérateurs mobiles (par exemple, le lien entre tarification, investissement technique, qualité de service, rentabilité) ;

- la dynamique de diffusion des innovations technologiques dans le temps et de la gestion des transitions technologiques (par exemple: GSM vers GPRS ; GSM vers UMTS) ;

- le jeu complexe liant concurrence et coopération entre opérateurs (guerre des prix ; interconnexion, couverture géographique des réseaux, MVNO, etc.).

Et tout ceci prend son sens en étant illustré par des exemples d'entreprises réelles de télécommunications mobiles au travers de leurs stratégies techniques, commerciales et managériales. L'introduction de l'UMTS, l'arrivée des MVNO, la couverture des «zones blanches » fournissent en France de telles illustrations.

Par ailleurs, le travail en équipes - souvent hétérogènes - oblige les participants à résoudre les questions classiques, mais difficiles, de la prise de décision en groupe et en temps limité.

\section{CONCLUSION}

La complexité des systèmes techniques comme les grands réseaux mobiles est considérable, et les enjeux économiques sont tels, qu'il est à la fois nécessaire et difficile de disposer d'outils de formation adaptés. Simobiz, jeu d'entreprise dédié à la simulation de marchés et réseaux mobiles, intègre les aspects techniques, économiques et managériaux et permet, dans un temps limité de relever ces défis. Comme tout jeu d'entreprise, dispositif ouvert, flexible et non-scolaire, il «plonge » les participants dans un univers industriel dans des conditions raisonnablement réalistes et stimule leur intérêt par l'émulation entre équipes gérant des entreprises concurrentes, la valorisation de la réflexion autonome et de la prise de décision. Dédié au monde des mobiles, il permet une réflexion approfondie sur les évolutions techniques et économiques de ce secteur.

\section{Bibliographie}

1.Hämmäinen H., Tallberg M. and Töyli J. A business management simulation of mobile service competition $9^{\text {th }}$ Workshop on Experimental Interactive Learning in Industrial Management (June 5-7, 2005, Espoo, Finland).
2. Kulich, R. Simulation de réseau mobile, Thèse de doctorat, Université de Zilina, Slovaquie, 2004.

3. Berne, M., Simobiz, Manuel du јеu, Tomes 1, 2, 3, INT, Evry, 2004.

4. http://www.telecom.gouv.fr/form/int_simobiz.htm

5.http://www.ituarabic.org/coe/Simobiz2/Invitation $\% 2$ 0-\%20Eng.doc 